\section{Ernestina Pérez Barahona (1865-1951): La medicina al rescate de la raza chilena}

\author{
HERNÁN SCHOLTEN ${ }^{1, a}$, GONZALO SALAS ${ }^{2, b}$, \\ JOSÉ RAMOS-VERA ${ }^{2, \mathrm{c}}$, JULIO CÉSAR OSSA ${ }^{3, \mathrm{~d}}$, \\ ELIZABETH PARDO-GONZÁLEZ ${ }^{2, \mathrm{e}}$
}

\section{Ernestina Pérez Barahona (1865-1951). Her medical attempt to rescue the chilean race}

This article analyzes some publications of Ernestina Pérez Barahona (18651951), the second Chilean and Latin American female physician. It exposes her concern for the public health and, more precisely, for the problem of the Chilean race. She constantly refers to hygiene in her conferences, articles and books published between 1887 and 1920. Thus, the aim of this review is to recover her production and to inform the readers about her intervention as a medical professional.

(Rev Med Chile 2020; 148: 387-392)

Key words: Chile; Hygiene; Physicians, Women.

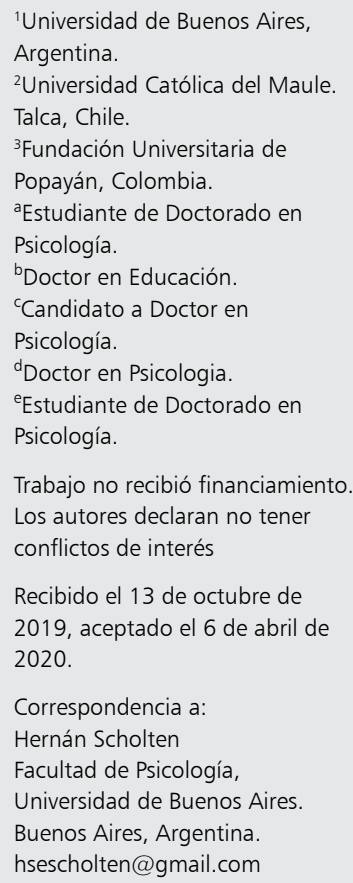

Correspondencia a: Hernán Scholten Facultad de Psicología, Universidad de Buenos Aires. Buenos Aires, Argentina. hsescholten@gmail.com

\section{L} a historia de la medicina es un campo de estudios que ha conocido un amplio desarrollo desde mediados del siglo XIX ${ }^{1}$ y que permite trazar los inicios de esta disciplina hasta la antigüedad $^{2}$. Su profesionalización es, sin embargo, mucho más reciente: en efecto, la mayor parte de las investigaciones coinciden al señalar la notable expansión de las carreras universitarias de medicina desde finales del siglo XIX en Occidente ${ }^{3,4}$.

El rol de las mujeres en el marco de esta historia dio lugar a interesantes análisis y amerita ser extensamente explorado. Como fue señalado por diversas autoras, en algunas civilizaciones antiguas se relacionaban las prácticas curativas con deidades femeninas, como Isis entre los egipcios y Panacea entre los griegos 5 . Más tarde, a comienzos de la Edad Moderna, las brujas fueron ampliamente relacionadas con prácticas curativas ${ }^{6}$ $y$, más recientemente, las mujeres se destacaron en su labor como curanderas y enfermeras ${ }^{7}$.

Desde el último tercio del siglo XIX, las mujeres occidentales pudieron ir conquistando su lugar en el ámbito académico y aspiraron a un título profesional. Así lo muestra el caso particular de la medicina, salvo algunos casos excepcionales como el de Dorothea Erxleben (1715-1762) que, en 1754, obtuvo su doctorado en la Universidad de Halle ${ }^{8}$. Elizabeth Blackwell (1821-1910) fue la primera mujer que recibió el título de médica en los EE. UU. en $1849^{9,10}$ y, veinticinco años más tarde, Rebecca Lee Crumpler (1831-1895) se transformó en la primera médica afroamericana ${ }^{11}$. En Europa, Elizabeth Garrett Anderson (1836-1917) fue la primera británica que obtuvo la licenciatura en medicina en $1865 \mathrm{y}$, diez años más tarde, Madeleine Brès (1842-1921), fue la primera doctora en medicina francesa ${ }^{10,12}$. Finalmente, las primeras médicas españolas fueron Martina Castells Ballespí (1852-1884) y Dolors Aleu i Riera (1857-1913), que consiguieron su doctorado en 1882, con apenas cuatro días de diferencia ${ }^{13}$.

En el caso de América Latina, interesa aquí destacar dos trabajos recientes que se concentran en las figuras de Eloísa Díaz Insunza (1866-1950) y Ernestina Pérez Barahona (1865-1951), las primeras médicas de la región. Mientras que el primero de estos textos propone esencialmente un cuadro comparativo de sus biografías ${ }^{14}$, el otro artículo se concentra en el enfoque higiénico que atraviesa diversas producciones de Díaz Insunza ${ }^{15}$. 
A la luz de estas producciones, el presente artículo tiene como objetivo recuperar y analizar algunas intervenciones y publicaciones de Pérez Barahona para dar cuenta de su conexión con el problema de la salud pública y, más precisamente, con el problema de la raza. Esto se hará visible a través de las múltiples referencias a la cuestión de la higiene en muchas de sus conferencias, artículos y libros, que permiten delinear su modalidad de intervención desde la profesión médica.

\section{Itinerario biográfico}

Si bien los estudios biográficos sobre Pérez Barahona son escasos y fragmentarios, permiten rescatar algunos datos relevantes de su vida ${ }^{16}$. Nacida el 8 de agosto de 1865 en la ciudad de Valparaíso, cursó sus estudios secundarios en el Liceo Isabel Le Brun de Pinochet en Santiago. Obtuvo el Bachillerato en Humanidades en 1883 y, apenas dos años después, consiguió el Bachillerato en Medicina y Farmacia. Finalmente, tras alcanzar su licenciatura el 31 de diciembre de 1886, recibió el título de médico-cirujano el 10 de enero de 1887, una semana después que lo obtuviera Eloísa Díaz Insunza. De este modo, se convertía en la segunda médica chilena y latinoamericana. Tras finalizar sus estudios, Pérez Barahona tuvo una destacada participación en el combate contra la epidemia de cólera que azotó a Valparaíso, lo cual le valió el título de ciudadana ilustre ${ }^{17}$.

Partió posteriormente a continuar sus estudios en Europa, becada por el Gobierno de Chile ${ }^{18}$, que gestionó un permiso especial para que pudiera asistir a la Friedrich-Wilhelms-Universität (actual Humboldt-Universität) en Berlín, ya que las mujeres tenían vedado el acceso a esa academia. Fue la primera médica chilena que realizó estudios en el exterior ${ }^{19}$ y la primera latinoamericana que ingresó en la academia prusiana, donde habría obtenido el reconocimiento local al ser integrada a la Academia Médica de Berlín. Continuó su formación en París y, cuando regresó a Chile en 1891, el presidente José Manuel Balmaceda le habría otorgado un puesto en el servicio de ginecología del Hospital San Borja. Tuvo una destacada participación en los congresos médicos latinoamericanos realizados en Santiago (1901) y Buenos Aires (1904), donde presentó diversas ponencias ${ }^{20}$.

Por último, es preciso al menos mencionar su participación en organizaciones femeninas tales como el Club de Señoras (1915), que agrupaba a las mujeres de la élite ilustrada de Santiago, y el Círculo de Lectura (1915). Como desprendimiento de esta última institución, en 1919 se creó el Consejo Nacional de Mujeres y Ernestina Pérez fue electa como su primera presidenta. Similar situación se presentó más tarde, en 1931, al ser electa como primera presidenta de la Asociación Nacional de Mujeres Universitarias de Chile, espacio que compartió con Amanda Labarca y Elena Caffarena ${ }^{21}$. Poco más tarde, se retiró de la actividad médica y falleció en su ciudad natal en 1951.

\section{El higienismo}

Más que analizar detalladamente la variedad de tópicos que Pérez Barahona fue abordando durante más de treinta años de producción intelectual, interesa aquí señalar una temática general que parece recorrer la totalidad de sus publicaciones y que, más allá de algunas coincidencias biográficas, ligan sus intervenciones con la producción de Díaz Insunza: el problema de la higiene ${ }^{15}$.

Para ello, es preciso recordar que la cuestión de la higiene se impuso paulatinamente en el ámbito de la medicina occidental desde finales del siglo $\mathrm{XVIII}^{22,23}$, cuando la prevención de las enfermedades comenzó a asumir un papel preponderante e impuso una nueva agenda y un creciente prestigio al médico profesional. Como lo muestran las indagaciones de Michel Foucault sobre la biopolítica, el surgimiento de la medicina social en el siglo XVIII $^{24}$ permite mostrar que la salud de la población se convirtió en una preocupación central de los estados occidentales, lo cual impulsó amplias reformas sociales que permitieron implementar algunas medidas profilácticas. Junto con la difusión de la vacuna durante el siglo XIX, es posible apreciar la amplia divulgación de los manuales de higiene desde el siglo XVIII ${ }^{25}$.

En el caso de Chile, la publicación de Catecismo Hijiénico o Arte de Conservar la Salud ${ }^{26}$, en 1859, permite fechar los inicios de la difusión del higienismo a nivel local. Durante las siguientes décadas, se editaron diversos manuales de higiene de autores locales y también se tradujeron textos extranjeros, especialmente franceses ${ }^{27}$. Entre los médicos, Adolfo Murillo fue uno de los difusores iniciales del higienismo a nivel local: en 1872, luego de que presentara un informe sobre la 
educación física y la higiene al gobierno chileno, se aprobó el decreto de enseñanza de la higiene en las escuelas. Otra figura destacada es Ricardo Dávila Boza, considerado un pionero de la infectología en Chile. En 1884, publicó "La hijiene de la escuela", en donde propuso, por primera vez, incluir el ámbito escolar en la agenda sanitaria local. La preocupación por los brotes epidémicos que sacudieron a Chile hacia finales del siglo XIX, impulsaron diversas medidas legislativas y de la creación de nuevas instituciones (por ejemplo, Consejo Superior de Higiene Pública y el Instituto de Higiene), que muestran el avance de las ideas higienistas en Chile hasta la sanción del primer Código Sanitario que, a comienzos del siglo XX, "transforma los principios del paradigma [higienista] en norma jurídica" 28,29 .

Los aportes de Ernestina Pérez Barahona a la difusión del higienismo en Chile, se aprecian claramente ya en su memoria de prueba que, si bien retoma el formato de ciertos manuales que se difundían en Chile desde la década de 1870, su título indica una perspectiva enfocada en el ámbito público: Elementos de hijiene popular ${ }^{30}$. La autora plantea desde un inicio la imperiosa necesidad de que las nociones de la ciencia higiénica se popularicen en Chile, lo cual no solamente permitiría que la población llegue incluso a triplicarse, sino que "la generación que se levante será fuerte y vigorosa" ${ }^{30}$. Le otorga entonces el rango de responsabilidad del Estado, en tanto correspondería a "nuestros gobernantes, velar por la salud del pueblo, haciendo obligatoria la enseñanza de la higiene en todas las escuelas de república, sobre todo en las de mujeres" ${ }^{30}$. La primera parte del texto se ocupa, con un tono marcadamente prescriptivo, de temáticas diversas -tabaco, el ejercicio, el sueño, la vivienda, la vestimenta, el aseo o limpieza, las enfermedades y vacunas-, dedicando un espacio destacado a la cuestión de los alimentos y de las bebidas, especialmente en el caso de los lactantes, que ocupan casi la mitad de este volumen.

La segunda parte de esta memoria de prueba contiene los aportes más originales de la autora. En efecto, más allá de los aspectos de la salud corporal, comunes en la literatura higienista, en esta sección se proponen, como su título lo señala, algunas indicaciones que apuntan a la higiene moral. En este sentido, comienza destacando el valor de la instrucción, una cuestión que está por fuera del ámbito estrictamente médico, como modo de ejercitar y ampliar el círculo de ideas del cerebro órgano cuyo desarrollo, señala el texto, marca la superioridad del hombre respecto de las demás criaturas. Además, como lo muestra la cita que se reproduce a continuación, señala su impacto en los vínculos familiares y sociales:

La cultura del espíritu desarrolla todas nuestras facultades (...), eleva el alma, desarrolla los bellos sentimientos, ennoblece en el corazón i dirije nuestras acciones en la vía del bien. Nos hace buenos, útiles i queridos de nuestros semejantes; estrecha los lazos con que están unidos los esposos i les hace dar una buena educación a sus hijos. En fin, la cultura del espíritu es la fuente de una multitud de dichas i placeres de los cuales el hombre ignorante queda ajeno ${ }^{30}$.

Por este motivo, la autora estima necesario añadir algunas directivas respecto de la elección de los libros infantiles, para evitar que los niños "se llenen la cabeza con ideas estravagantes", y respecto de las supersticiones que "están en razón directa del grado de cultura e ilustración del pueblo" ${ }^{30}$. Asimismo, condena los juegos de azar y promueve tanto el ahorro como los paseos campestres, destacando sus beneficios para la salud.

De este modo, Ernestina Pérez Barahona indagaba, en su memoria de prueba, numerosos y diversos aspectos de la vida humana para proponer, inspirándose en los principios científicos de la higiene, directivas que permitan su normalización o regulación desde un abordaje claramente profiláctico a nivel social.

Las referencias a la higiene se multiplican en sus posteriores publicaciones, ya sea desde sus títu$\operatorname{los}^{31} \mathrm{o}$ a partir de la difusión, en el ámbito médico local, de los saberes sobre la salud femenina elaborados en Europa ${ }^{32}$. Este espíritu divulgador está mucho más claramente presente en su Manual de la enfermera en el hogar, que establece la relevancia y jerarquía de la higiene en las dos frases iniciales de su prólogo: tras el refrán "Más vale prevenir que curar", la autora afirma que "[l] a higiene es una ciencia mucho más provechosa que la medicina" ${ }^{33}$. Y si bien se trata de un texto dirigido a la mujer chilena, especialmente en sus roles de madre y maestra, sigue apuntando, como lo hacía ya desde su memoria de prueba, a la sociedad, a la preservación de su salud y defensa.

Como se verá a continuación, esta última cuestión asumirá un perfil más nítido en su producción poco tiempo más tarde, hacia finales de la década de 1910. 


\section{La raza chilena}

En 1917, Pérez Barahona dictó una conferencia sobre los microbios en el Club de Señoras, la cual motivó que Arturo Alessandri, presente entre el público y por ese entonces Senador, acelerara la aprobación del Código Sanitario (1918). Con este precedente, tres años más tarde, en el mismo lugar, presentó otra conferencia que, desde un enfoque explícitamente higienista, proponía mostrar las consecuencias del consumo cotidiano de las bebidas alcohólicas y apoyar la campaña antialcohólica anunciada por el propio Alessandri, ahora Presidente de la República. Este objetivo es alineado explícitamente en un programa que, según la autora, buscaba "salvar a la raza chilena" ${ }^{34}$.

De este modo, establecía claramente como telón de fondo de sus propuestas el problema de la raza, cuya discusión se inició en Europa y que comenzó a tematizarse en Chile hacia finales del siglo XIX. En 1904, se publicó Raza chilena ${ }^{35}$ de Nicolás Palacios, primer tratado sistemático sobre este tópico a nivel local y que, más allá de sus diversas e insostenibles tesis sobre sus orígenes y características, ilumina una polémica que tuvo sus variantes locales en la mayor parte de las jóvenes naciones latinoamericanas, y que se extenderá al menos hasta la década de $1950^{36}$.

En el caso de Ernestina Pérez Barahona, más que una discusión sobre las características de la raza chilena, su Conferencia muestra una preocupación estrictamente médica en torno a la salud y la supervivencia de la población local. En efecto, la decadencia de la raza no remite aquí a ciertos ideales de pureza o a la preocupación por el mestizaje, sino que se evidencia a partir de la elevada mortalidad infantil, que contrasta notoriamente con el panorama presente en "países civilizados" como Francia, Inglaterra, Suecia, Noruega, etc.: "esta raza chilena", afirma Ernestina Pérez Barahona, "desaparecerá en un tiempo más o menos corto, si no combatimos las causas de esta espantosa mortalidad"34. Entre estas causas, tanto entre los ricos como entre los pobres, señala en primer lugar al alcoholismo.

Es desde esta perspectiva que debe entenderse que la cuestión de la raza sea aquí abordada a partir del problema de la degeneración y la herencia, como lo muestra la caracterización del alcohol como "demonio de la degeneración" que altera los órganos y células, y las reiteradas referencias al he- redo-alcoholismo. Apoyándose en los estudios del médico francés Jules Hericourt (1850-1938), sostiene que tanto el delirium tremens, la dipsomanía, como también la degeneración física, intelectual y moral, pueden transmitirse a los hijos. De hecho, los efectos de la heredo-degeneración resultarían ya apreciables en la población carcelaria y manicomial, así como en el caso de los araucanos. En tanto un futuro similar se aproximaría para la raza chilena, Pérez Barahona entiende que es preciso atacar las causas que incitan al alcoholismo, entre los cuales señaló diversos factores psicológicos y sociológicos ${ }^{34}$.

Respecto de las medidas preventivas, el texto se propone esencialmente exhibir las ventajas de la supresión del alcohol, tanto a nivel individual como social. En efecto, así como las personas sobrias, e incluso las que beben ocasionalmente, "favorecen con esto su salud, la fuerza de su inteligencia y la vejez se retarda", se afirma que la raza chilena, librada del vicio del alcohol, es superior "a muchos otros pueblos del mundo por su energía física y por su inteligencia" 34 . En este último caso, refiere tanto al exitoso régimen que se implementó en las tropas del norte de Chile como a los efectos de la ley seca en los EE. UU., que la autora presenta como modelo a seguir a nivel local: “¿Qué ejemplo más digno de imitar! ¡tengo la intuición de que los chilenos lo seguiremos, guiados por un grande $y$ patriótico cerebro!"34.

\section{Conclusión}

Las temáticas expuestas a partir de esta conferencia sobre el alcoholismo, sirven como guía para bosquejar una lectura retrospectiva de la producción intelectual de Pérez Barahona, en tanto permiten iluminar con mayor claridad la insistencia de algunos elementos en la mayor parte de sus publicaciones, mencionadas anteriormente. En efecto, sea que se aborde cuestiones de ginecología, sobre la salud de la mujer (especialmente en su rol materno) o sobre la infancia, se trata de formular indicaciones relativas a la salud de la población. Y esta preocupación por la higiene popular, ya presente desde muy temprano en su producción $^{31}$, es considerada como una herramienta fundamental para salvaguardar a la raza chilena, cuyo porvenir estaría amenazado por los fantasmas de la degeneración y la herencia. 
Ahora bien, aunque este análisis revele un aspecto notable de la obra de Pérez Barahona, de ningún modo llega a configurarse como un estudio exhaustivo de su producción intelectual. En este sentido, se busca aquí señalar algunos tópicos que permitan iniciar nuevas investigaciones de su trayectoria e indaguen aspectos hasta ahora escasamente explorados. En efecto, su vida y su obra constituyen un valioso capítulo en la historia de la medicina y especialmente de la ginecología y la pediatría no solamente en Chile sino a nivel regional. Por otra parte, sus intervenciones y publicaciones deben ser contempladas como un capítulo en la historia de las mujeres.

Finalmente, como prolongación de este mismo artículo, y retomando algunas de las ideas aquí sugeridas, es posible ubicar con mayor precisión los aportes de esta médica chilena a luz de las investigaciones que se están realizando sobre la cuestión del higienismo y la raza en Chile.

\section{Referencias}

1. Eslava J. La historia de la historia de la medicina: una exploración inicial (Primera parte). Revista Colombiana de Filosofía de la Ciencia 2014; 14 (1): 147-173. Disponible en https://www.redalyc.org/pdf/414/41431645007. pdf

2. Magner LN. A history of medicine. Boca Raton: Taylor \& Francis Group, 2005.

3. Thistlethwaite J, Spencer J. Professionalism in Medicine. Florida, EE. UU.: CRC Press, 2008.

4. Tomkins A. Medical Misadventure in an Age of Professionalisation, 1780-1890. Manchester: Manchester University Press, 2017.

5. Wynn R. Saints and Sinners: Women and the Practice of Medicine Throughout the Ages. JAMA 2000; 283 (5): 668-9.

6. Windsor L. Women in medicine. An Encyclopedia. Santa Barbara: ABC-CLIO, 2002.

7. Perrone B, Stockel H, Krueger V. Medicine Women, Curanderas and Women Doctors. Oklahoma: University of Oklahoma Press, 1989.

8. Goddemeier C. Dorothea Christiane Erxleben: Doktor der “Arzneygelahrtheit”. Deutsches Arzteblatt International 2013; 110 (17): A841. Disponible en https://www. aerzteblatt.de/archiv/137624/Dorothea-Christiane-Erxleben-Doktor-der-Arzeneygelahrtheit.

9. Ellis H. Elizabeth Blackwell: a pioneer female medical graduate. British Journal of Hospital Medicine 2010; 71
(5): 281-281. Disponible en https://www.magonlinelibrary.com/doi/abs/10.12968/hmed.2010.71.5.47909.

10. Toubas P. L. Historical perspectives: Pioneer woman in neonatology: Part 1: 1870-1945. NeoReviews 2011; 12 (5): 247-51. Disponible en http://hw-f5-neoreviews. highwire.org/content/12/5/e247.

11. Gates HL, Higginbotham EB. African American Lives. Oxford: Oxford University Press, 2004.

12. Cavendish R. Britain's first female doctor: Elizabeth Garrett Anderson passed her medical exams on September 28th 1865. History Today 2015; 65 (9). Recuperado de https://www.historytoday.com/archive/months-past/ britain $\%$ E2\%80\%99s-first-female-doctor.

13. Flecha García C. Women's education according to the first women to receive doctorates in medicine from Spanish universities, 1882. Dynamis 1999; 19 (1): 241-78.

14. Osorio Abarzúa CG. Las primeras médicas de Chile y Latinoamérica: Eloísa Díaz Inzunza y Ernestina Pérez Barahona. Rev Med Chile 2019; 147 (3): 367-71. Disponible en https://scielo.conicyt.cl/scielo.php?script=sci_arttext\&pid=S0034-98872019000300367.

15. Salas G, Scholten H, Guerra-Labbé L, Ramos-Vera J, Pardo-González E. Eloísa Díaz Inzunza: entre la medicina, la psicología y la educación. Rev Med Chile 2019; 147 (4), 499-504. Disponible en https:// scielo.conicyt.cl/scielo.php?script=sci_arttext\&pi$\mathrm{d}=$ S0034-98872019000400499.

16. Unión Chilena de Mujeres. Homenaje a la doctora Ernestina Pérez Barahona. Santiago: Ediciones de la Unión Chilena de Mujeres, 1953. Disponible en http://www. memoriachilena.gob.cl/602/w3-article-9125.html.

17. AA. VV. Las primeras mujeres que abrieron camino en Chile. Santiago: Ediciones El Mercurio, 2019.

18. Anónimo. Las primeras mujeres médicas en Chile. Anales Chilenos de Historia de la Medicina 1967-1968; IX-X (1): 13-16. Disponible en http://www.bibliotecaminsal. cl/wp/wp-content/uploads/2013/05/anales_anos910_vol1_p13.pdf.

19. Zárate Campos M. S. Al cuidado femenino. Mujeres y profesiones sanitarias, Chile, 1889-1950. En Stuven A. M., Fermandois J. (Ed). Historia de las mujeres en Chile II. Santiago: Taurus/Aguilar Chilena, 2013, pp. 119-155. Disponible en https://www.academia. edu/6369861/_Al_cuidado_femenino._Mujeres_y_profesiones_sanitarias_1889-1950_en_Ana_Maria_Stuven_y_Joaqu\%C3\%ADn_Fermandois_editores_Historia_de_las_mujeres_en_Chile_Tomo_II_Santiago_2013

20. Segundo Congreso Médico Latino Americano. Actas y Trabajos. Buenos Aires: Imprenta i Editora de Coni Hermanos, 1905.

21. Pardo A. Historia de la mujer en Chile. La conquista 
de sus derechos políticos en el siglo XX (1900-1952). Disponible en https://critica.cl/historia/historia-de-lamujer-en-chile-la-conquista-los-derechos-politicosen-el-siglo-xx-1900-1952. Consultado el 21 de julio de 2019.

22. Vigarello G. Histoire des pratiques de santé. Le sain et le malsain depuis le Moyen Age. París: Seuil, 1999.

23. Smith V. Clean. A history of personal hygiene and purity. Oxford: Oxford University Press, 2007.

24. Foucault M. Nacimiento de la medicina social. En Obras esenciales II. Madrid: Paidós Básica, 1999, pp. 363-84.

25. Moreno Martínez P. Presentación. Cuerpo, higiene, educación e historia. Historia de la educación 2009; 28 (1): 23-36. Disponible en http://revistas.usal.es/index. php/0212-0267/article/viewFile/10260/10693.

26. Miquel J. Catecismo Hijiénico o Arte de Conservar la Salud. Santiago: Imprenta del Ferrocarril, 1859.

27. Folchi Donoso M. La higiene, la salubridad pública y el problema de la vivienda popular en Santiago de Chile, 1843-1925. En Loreto López R, coord. Perfiles habitacionales y condiciones ambientales: historia urbana de Latinoamérica, siglos XVII-XX. Puebla: BUAP, 2007, pp. 361-88.

28. Ministerio del Interior. Ley $\mathrm{N}^{\circ} 3385$. Código Sanitario. Santiago: Imprenta Central, 1918.

29. Simón Pérez I, Sánchez Andaur R. Introducción del paradigma higiénico sanitario en Chile (1870-1925): discursos y prácticas. Anuario de Estudios Americanos 2017; 74 (2): 643-74. Disponible en http://estudiosamericanos.revistas.csic.es/index.php/estudiosamericanos/ article/view/723.

30. Pérez Barahona E. Elementos de higiene popular. Santiago: Facultad de Medicina i Farmacia, Universidad de Chile, 1887. Disponible en http://www.museomedicina. cl:8080/bitstream/handle/123456789/2883/MD0008002. pdf.

31. Pérez Barahona E. Resumen de consejos higiénicos aplicados especialmente a la infancia. Santiago: Imprenta de San José, 1910.

32. Pérez Barahona E. Lecciones de ginecología. Berlín, s. n, 1910.

33. Pérez Barahona E. Manual de la enfermera en el hogar. Santiago: Imprenta Universitaria, 1918.

34. Pérez Barahona E. Conferencia sobre el alcoholismo dada en el Club de Señoras por la doctora Ernestina Pérez. Santiago: Prensa Universitaria, 1920. Disponible en http://www.memoriachilena.gob.cl/602/w3-article-9130. html.

35. Palacios N. Raza Chilena. Santiago: Imprenta i Litografía Alemana de Gustavo Schafer, 1904.

36. Gutiérrez H. Exaltación del mestizo: La invención del Roto Chileno. Universum 2010; 25 (1): 122-39. 